\title{
Aqueous Nanofluids Based on Copper MPA: Synthesis and Characterization
}

\author{
Caio Carvalho dos Santos ${ }^{a *}$, Wesley Renato Viali ${ }^{a}$, Eloiza da Silva Nunes ${ }^{a}$, Douglas Ricardo de Assis ${ }^{a}$, \\ Bruno Estevan Amantéa ${ }^{a}$, Miguel Jafelicci Júnior ${ }^{a}$
}

${ }^{a}$ Laboratório de Materiais Magnéticos e Colóides, Departamento de Físico Química, Instituto de Química, Universidade Estadual Paulista, Araraquara, SP, Brazil

Received: March 22, 2017; Revised: October 24, 2017; Accepted: November 27, 2017

\begin{abstract}
The application and use of efficient cooling fluids have become increasingly important due to the increasing industrial and energy demand associated with the miniaturization of various electronic devices. The search for high-efficiency heat exchanger fluids in the early 1990 s led to the development of a new class of refrigerants called nanofluids. The use of nanofluids is linked to obtaining stable colloidal dispersions which exhibit high thermal conductivity. For this purpose, the efficiency of a nanofluid will depend on the type of fluid used and the dispersed nanomaterial. In this work, a stable aqueous nanofluid based on mercaptopropionic acid-coated copper sulfide nanoparticles $\left(\mathrm{Cu}_{2} \mathrm{~S} / \mathrm{MPA}\right)$, synthesized by the chemical reduction method, was developed. The nanofluid presented colloidal stability in alkaline medium and an average increase of $36 \%$ in thermal conductivity for a volumetric fraction of $0.05 \%$.
\end{abstract}

Keywords: Nanofluids, copper sulfide, thermal conductivity, and mercaptopropionic acid.

\section{Introduction}

The increasing demand for heat flows and energy loads in many technological areas, such as electronics, transportation, and data center servers, requires more efficient heat dissipation to ensure operational use and extend the lifetime of refrigerated components. ${ }^{1-6}$ The use of conventional refrigeration fluids has been enhanced to develop more efficient heat exchanger fluids. In this context, in the early 1990s, a new material class was conceived. These new materials were more efficient than liquid refrigeration and became known as nanofluids..$^{7-10}$ The interest in the development of more efficient refrigeration methods has caused an increase in the number of studies that have been reported in nanofluid tests. Indeed, in a review by Babu et al 2017, it is affirmed that in the USA in 2008 the use of nanofluids in the energy industry saved 10-30 trillion Btu of energy during the course of the year. ${ }^{8}$

Nanofluids are composites formed by the stable colloidal dispersion of nanomaterials (with a size less than $100 \mathrm{~m}$ ) in a conventional cooling fluid. ${ }^{3-11}$ Conventional fluids used in liquid refrigeration, such as water, ethylene glycol and oils, have low thermal conductivity (thermal conductivity of water $0.613 \mathrm{~W} / \mathrm{m} \cdot \mathrm{K}$ at $300 \mathrm{~K}$ ), while solid materials, on the other hand, have higher thermal conductivity values. Due to the high thermal conductivity of solids, the addition of nanomaterials (such as ceramics, metals, alloys, semiconductors and carbon derivatives) is a way of increasing the thermal conductivity of conventional fluids, resulting in a fluid composite with higher heat exchange efficiency. ${ }^{6,12,13}$

*e-mail: caio.cvs@hotmail.com
The addition of nanomaterials to conventional refrigeration fluids in small volumetric fractions produces systems with higher colloidal stability when compared to micrometric systems. In addition, nanomaterials can flow smoothly without causing clogging of refrigeration systems and provide improvements in the thermal properties of the fluid. ${ }^{9-12}$

The key issue to obtaining efficient nanofluids is to synthesize nanomaterials that have appreciable colloidal stability by modifying or functionalizing their surfaces. Aiming to produce nanofluids with higher colloidal stability in recent years, several materials have been studied as surface agents of nanoparticles, such as polymers, surfactants, aminosilanes and other multifunctional molecules. ${ }^{14-16}$

Nanofluids are produced by two different routes, designated as a one-step method and a two-step method. ${ }^{4-12}$ In the onestep method, the nanomaterials were synthesized directly in a conventional cooling fluid, forming the nanofluid. The major advantage of this method is to avoid the formation of nanoparticle agglomerates, which favors the colloidal stability of the nanofluids. However, most of the synthetic routes used in the one-step method require materials that have low vapor pressure and are usually very expensive. Chemical routes have been proposed for a wide variety of materials, but in this method the impurities of the synthesis remain in the final product, which affects the quality of the nanofluid. ${ }^{17-22}$

In the two-step method, the nanomaterial is first synthesized, washed and then dispersed in the conventional fluid to form nanofluid. This is the most widely used method because it is cheaper and allows high-scale use. The main difficulty of this 
method is related to the agglomeration of the nanomaterials. To overcome this problem, surface agents and mechanical agitation or ultrasound are used to break these aggregates and produce nanofluids with high colloidal stability. ${ }^{22,23}$

Recent works have addressed the use of nanoparticles based on metallic copper and copper oxides and sulfides. Wilk et al 2017 published an experimental paper that analyzed the thermal conductivity of uncoated $40 \mathrm{~nm}$ copper spherical nanoparticles in the 0.101 volumetric fraction and observed an increase in thermal conductivity of $11.5 \%$ at $23^{\circ} \mathrm{C} . .^{24}$ Xuan et al, in 2000 , observed increases of 2.5 to $7.5 \%$ in the thermal conductivity of the nanofluids with nanoparticles of suspended copper in water in the volumetric fractions of 1.24 to $1.78 .{ }^{25}$ Then in 2006 Liu et al published a result of copper nanofluid in uncoated water at the $1 \%$ volumetric fraction in which the conductivity of the nanofluid increased by $23.8 \% .{ }^{26}$ In 2010 Wei et al reported a study using CuS/ $\mathrm{Cu}_{2} \mathrm{~S}$-based nanofluids at different concentrations and observed oscillations in the thermal conductivity of the nanoparticles produced. ${ }^{27}$ Pryazhnikov et al 2017 published a paper where they evaluated the thermal conductivity of different oxide-based nanofluids as a function of size and volumetric fraction. The conclusions of their study observed that many factors affect the thermal conductivity of nanofluids, not only concentration of the nanoparticles, but also the size, type of material and type of base fluid. ${ }^{28}$ Therefore, the results described in the literature are highly dissimilar, since in many studies the purity and chemical and colloidal stability of the materials used are not clear and hinder the correlation of different publications.

In the present work, a copper sulfide-based nanofluid with high thermal conductivity was synthesized by the two-step method. Firstly, $\mathrm{Cu}_{2} \mathrm{~S}$ nanoparticles were synthesized by the chemical reduction method in the presence of mercaptopropionic acid (MPA) to obtain the nanomaterial. After synthesizing the nanoparticles, they were washed and then dispersed in distilled water in order to obtain the nanofluid.

\section{Experimental}

\subsection{Materials}

All the chemical reagents and solvents used in the work presented analytical grade and were used without any purification. For the synthesis, Argon (Ar) was used as purge gas. Sodium borohydride ( $96 \%$ Fluka), copper nitrate trihydrate (98\% Sigma Aldrich), triethylene glycol (99\% Sigma Aldrich), mercaptopropionic acid (>99\% Sigma Aldrich), ethanol (99.5\% Synth) and acetone (99.5\% Synth).

\subsection{Methods: synthesis of $\mathrm{Cu}_{2} \mathrm{~S}$ nanoparticles and obtaining nanofluid}

The copper sulfide nanoparticles were synthesized by chemical reduction method..$^{3,29-31}$ The procedure performed was the dissolution of $2 \mathrm{mmol}$ of copper nitrate in $20 \mathrm{~mL}$ in triethylene glycol. The solution was placed in a three-necked flask and left under magnetic stirring under argon atmosphere for 30 minutes at a temperature of $55^{\circ} \mathrm{C}$. Then $140 \mu \mathrm{L}$ of mercaptopropionic acid was added to the synthesis. After 10 minutes of reaction, $6 \mathrm{mmol}$ of sodium borohydride were injected to the synthesis under stirring for 10 minutes, and the temperature was adjusted to $140{ }^{\circ} \mathrm{C}$ for 120 minutes. The nanoparticles were washed twice with ethanol and once with acetone, the obtained material was dispersed in $30 \mathrm{ml}$ of distilled water to form the aqueous $\mathrm{Cu}_{2} \mathrm{~S}$ nanofluid, and this was left under mechanical stirring ( $3 \mathrm{rpm})$ for 7 days to stabilize.

\subsection{Characterization}

The X-ray powder diffraction (XRD) of the samples was recorded in the $2 \theta$ range of 20 to $50^{\circ}$ with rate $0.02 \%$ using the Siemens D5005 system equipped with a $\mathrm{Cu}$ $\mathrm{K} \alpha(\mathrm{I}=1.5418 \AA)$ radiation source with a voltage of $40 \mathrm{KV}$ and current of $40 \mathrm{~mA}$.

The FTIR measurements were carried out in a PerkinElmer Frontier Dual Range FTIR spectrometer using the system resolution set at $4 \mathrm{~cm}^{-1}$ while performing 28 scans using potassium bromide $\mathrm{KBr}$ pellets (approximately 1:99, at a pressure of about 10 bar of the pellet) in the region of 4000 to $400 \mathrm{~cm}^{-1}$.

DSC analysis was performed on a NETZSCH DSC 404 F3 Pegasus. The temperature range varied between $25^{\circ} \mathrm{C}$ and $600{ }^{\circ} \mathrm{C}$ with a heating rate of $10^{\circ} \mathrm{C} \mathrm{min}^{-1}$ and $\mathrm{N}_{2}$ atmosphere $\left(50 \mathrm{~mL} \cdot \mathrm{min}^{-1}\right)$. The mass used was approximately $33.6 \mathrm{mg}$ in closed and sealed aluminum crucibles and the empty capped crucible was taken as reference.

The Zeta potential $(\zeta)$ and dynamic light scattering (DLS) were determined in a Zetasizer Nano ZS, Malvern. The zeta potential was determined using 20 microliters of fluid to each milliliter of sodium chloride electrolyte, and the samples were titrated in the range of 7 to 11.5 measured with 0.5 $\mathrm{pH}$ step, measured in triplicate at $25^{\circ} \mathrm{C}$. The zeta potentials were obtained from the triplicate of measurements, in which the average and the standard deviation were calculated from electrophoretic movement measurements performed using phase analysis light scattering. Measurement of dynamic light scattering (DLS) was performed in triplicate polystyrene cuvettes at a temperature of $25^{\circ} \mathrm{C}$ using a He-Ne laser $(\lambda$ $=633 \mathrm{~nm}$ ) with a dispersion angle of $173^{\circ}$.

Transmission Electron Microscopy (TEM) images were obtained in a JEOL 3010 TEM-HR operating at $300 \mathrm{kV}$. The samples were dispersed in isopropanol in an ultrasound bath and then dropped onto a copper grid coated with carbon film. The measurements of high-resolution images were analyzed by Fourier transform (FFT) using the software "Digital Micrograph (Gatan)". To calculate the mean diameter at least 150 particles were counted using "ImageJ" software. 
The thermal conductivity of the nanofluid was measured by the hot wire method, which is based on heating a probe that functions as a linear heat source within the fluid to be evaluated. This method is widely used to measure the conductivity of nanofluids because it minimizes natural convection due to its relative simplicity. ${ }^{32}$ The measurement was performed with a TP-08 model probe, the temperature was equilibrated using a cooling bath, and the temperature was measured using a mercury bulb thermometer. Five measurements were made for each temperature in the range of 10 to $60{ }^{\circ} \mathrm{C}$. The probe was calibrated using glycerin at all temperatures analyzed, which is the fluid with known thermal conductivity. After the calibration of the probe, the data were obtained based on five measurements carried out in the nanofluid with a time interval of 600 seconds between the measures, to achieve thermal stabilization of the system between each measurement. Based on these five measurements, the average and the standard deviation of the thermal conductivity for the nanofluid were calculated at each of the temperatures analyzed. The uncertainty value provided by the manufacturer is $\pm 3 \%+0.02 \mathrm{~W} / \mathrm{m} \cdot \mathrm{K}$, so that for the measurements performed in the range of 10 to $40^{\circ} \mathrm{C}$ the observed standard deviation was less than $3 \%$. Even for the temperatures of 50 and $60^{\circ} \mathrm{C}$ these were of 4.8 and $9.0 \%$, and they can provide overestimated values of the thermal conductivity due to the contribution of convective effects of the nanofluid.

\section{Results and Discussion}

\subsection{Nanoparticle Characterization}

The X-ray diffractogram in Figure 1 shows the crystalline pattern of the synthesized nanoparticles. The diffractogram presents broadening peaks that refer to a low crystallinity of the material and are also associated with the nanometric size of the synthesized material. Based on this, two crystalline phases were identified; $\mathrm{CuS}$ JPCDS 6-464 and $\mathrm{Cu}_{2} \mathrm{~S}$ JPCDS 83-1462, of which the major part is $\mathrm{Cu}_{2} \mathrm{~S}$. As is known in the literature, the synthesis of this type of material is common to form multivalence compounds as a function of the synthetic conditions. $^{33,34}$

Figure 2 presents the transmission micrograph of the sample, and these images allow us to confirm the nanometric size and shape of the synthesized material. The nanoparticles were nearly spherical, and they interact strongly with each other by the mercaptopropionic layer. In Figure 2 (b) crystalline planes with a lattice spacing of $0.1967 \mathrm{~nm}$ are observed, very close to the plane (6 30 ) of $\mathrm{Cu}_{2} \mathrm{~S}$ JPCDS 8314-62.

Electron transmission micrograph particle counting was also performed to determine the average diameter of the copper sulfide nanoparticles, and the calculated mean diameter was $5.2 \pm 1.6 \mathrm{~nm}$.

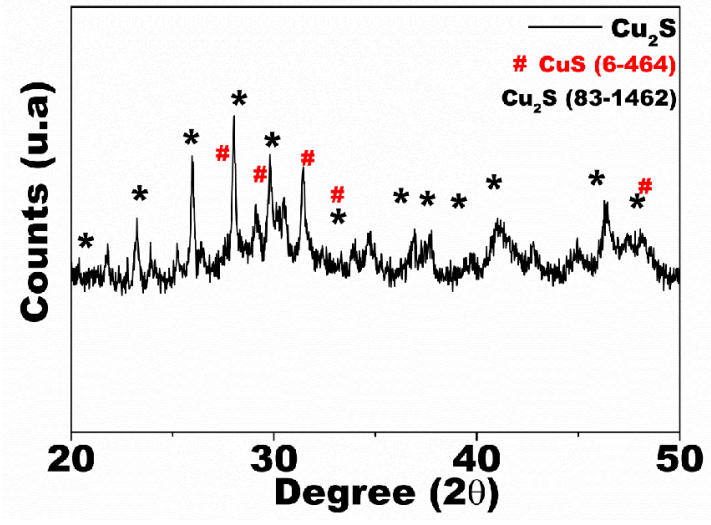

Figure 1. X-ray diffraction pattern of $\mathrm{Cu}_{2} \mathrm{~S}$ nanoparticles
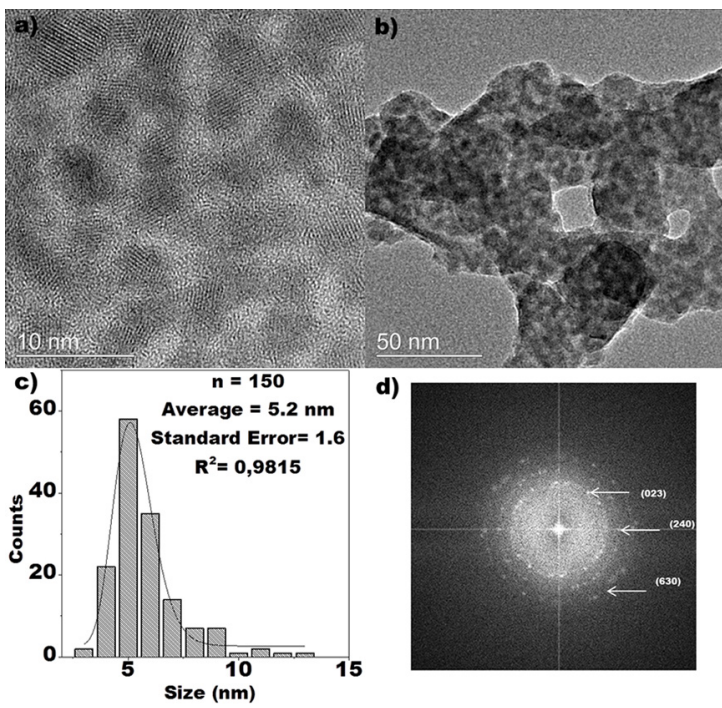

Figure 2. Transmission micrograph of $\mathrm{Cu}_{2} \mathrm{~S}$ sample: a) Highresolution and b) low magnification c) histogram of the nanoparticle count d) Fourier transform

The coating of nanoparticles was investigated by FTIR infrared spectroscopy. Figure 3 shows the infrared spectra of the precursor used in the MPA coating and the spectrum of the synthesized nanomaterial.

The bands in the 2665.1 and $2572.5 \mathrm{~cm}^{-1}$ regions of the S-H stretching were present in the MPA sample and absent in the $\mathrm{Cu}_{2} \mathrm{~S}$ sample, confirming the binding of MPA to the metal by the thiol group. The bands in the region of 3430.7 $\mathrm{cm}^{-1}$ were attributed to the $\mathrm{OH}$ stretching in the sample, while the band at $2935.6 \mathrm{~cm}^{-1}$ was attributed to the asymmetrical and symmetrical stretching of the $\mathrm{C}-\mathrm{H}$ bonds. The bands in the region of $1571.2 \mathrm{~cm}^{-1}$ to the $1427.5 \mathrm{~cm}^{-1}$ regions were attributed to the asymmetrical and symmetrical stretching of $\mathrm{COO}^{-}$group. ${ }^{35-37}$ Calculating the difference, delta $(\Delta)$, between the asymmetrical and symmetrical stretching bands yields a value of $143.7 \mathrm{~cm}^{-1}$ which is known in the literature as the ionic delta of some carboxylates, consistent with the $\mathrm{pH}$ of the sample. ${ }^{38}$ 


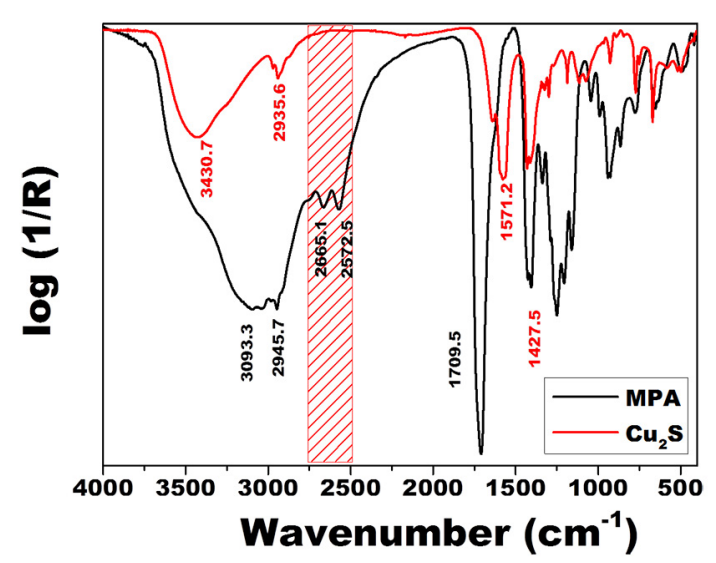

Figure 3. Infrared spectra of the $\mathrm{Cu}_{2} \mathrm{~S}$ and MPA sample.

In Figure 4 we observed the DSC curve of the sample in a nitrogen atmosphere to show possible phase changes.

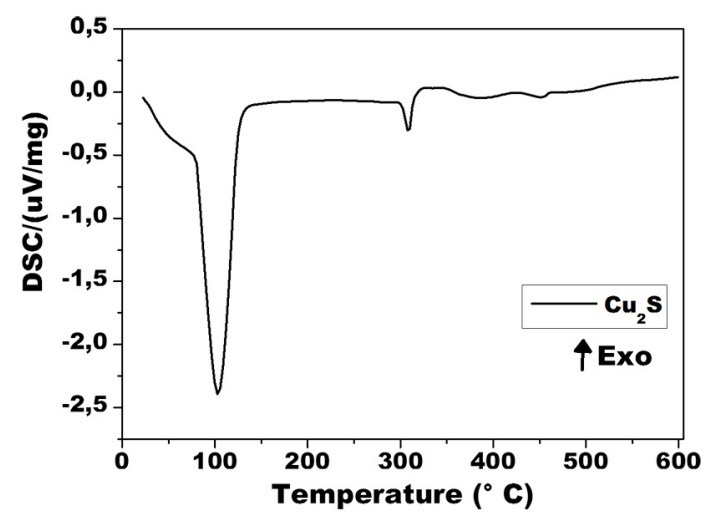

Figure 4. DSC of the $\mathrm{Cu}_{2} \mathrm{~S}$ sample.

The sample presents two endothermic thermal events that occur at 100 and $300^{\circ} \mathrm{C}$ respectively. The first one was correlated to the mass loss of adsorbed water and organic materials. The second was attributed to degradation of the bonded organic matter on the surface of the nanoparticles. ${ }^{39}$

\subsection{Nanofluid Characterization}

An aliquot of the sample was taken to determine the nanomaterial concentration in the nanofluid, and the analysis was performed by the technique of flame atomic absorption spectrophotometry. It was determined that the concentration of copper in the nanofluid was $2.28 \mathrm{~g} \mathrm{~L}^{-1}$. Based on the concentration, the volumetric fraction of the nanoparticles in the nanofluid was calculated, considering that the density of $\mathrm{Cu}_{2} \mathrm{~S}$ equal to $5.6 \mathrm{~g} / \mathrm{cm}^{3}$ obtained a volumetric fraction of approximately $0.05 \%$.

To complement the characterization of the sample, the Zeta-potential measurement of the sample was performed as a function of $\mathrm{pH}$ (Figure 5). The titration of the sample was performed from basic $\mathrm{pH} 11.5$ to neutral $\mathrm{pH}$, but acid
$\mathrm{pH}$ was not analyzed due to the instability of the sample in acid medium. ${ }^{40,41}$ The experimental points were fitted by the Boltzmann equation and are shown in Figure 5.

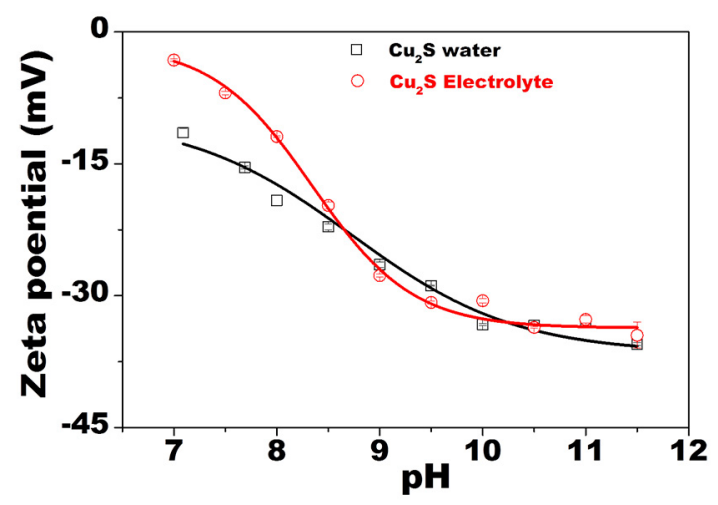

Figure 5. Zeta potential curves $(\zeta)$ as a function of $\mathrm{pH}$ for $\mathrm{Cu}_{2} \mathrm{~S}$ sample in water and $0.001 \mathrm{~mol} \mathrm{~L}^{-1} \mathrm{NaCl}$ electrolyte. In the graph the points correspond to the experimental data, and the line presents the fitting using the Boltzmann equation.

The sample was titrated as $\mathrm{pH}$ function in distilled water and $\mathrm{NaCl}$ electrolyte $0.001 \mathrm{~mol} \mathrm{~L}^{-1}$ to confirm the zeta potential curve profile of the synthesized nanomaterial. The analysis of the curves shows that the material produced has a considerable zeta potential value at basic $\mathrm{pH}$ greater than $9(\zeta>|25(\mathrm{mV})|)$. Also, the isoelectric point of the sample in the titrated range was not observed, which shows that the material can be used in this $\mathrm{pH}$ range. It is more stable at $\mathrm{pH}$ values between 9 and 10.5..$^{42}$ The hydrodynamic diameter of the nanoparticles was investigated at $\mathrm{pH} 9$, as can be seen in Figure 6.

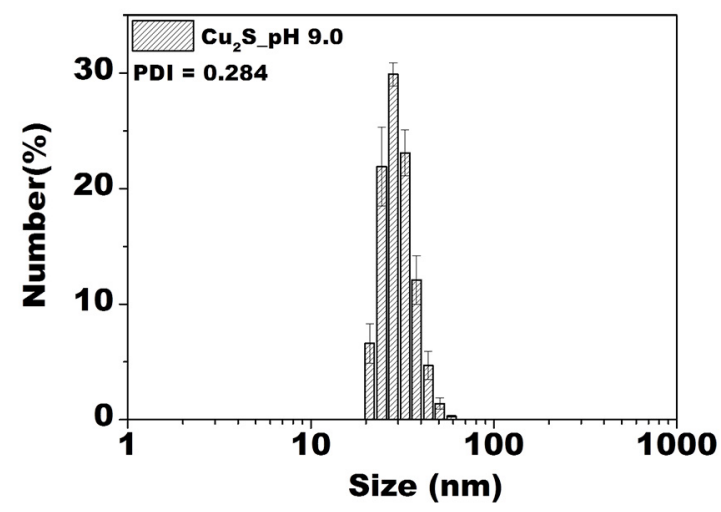

Figure 6. The hydrodynamic diameter of $\mathrm{Cu}_{2} \mathrm{~S}$ sample (DLS) by number.

Based on the data obtained by the DLS, the sample is polydisperse and shows a high polydispersity index (PDI $=0.284$ ), indicating the presence of aggregates. These may be due to the strong interactions between the MPA and the nanoparticles forming organized structures, such as observed 
in TEM. Although there are aggregates, their concentration in the sample is almost negligible, as can be observed from the DLS graph. A statistical number of the sample is formed by nanoparticles with a hydrodynamic diameter in the range of $30 \mathrm{~nm}$.

The thermal conductivity of the nanofluid synthesized by the hot wire method ${ }^{43,44}$ was evaluated using a probe previously calibrated with glycerin. To quantify the increase in the thermal conductivity of the nanofluid, the measurement was made in distilled water (base fluid) and then in the nanofluid for each of the temperatures, correcting the values based on the calibration constants obtained. The increases in the thermal conductivity of nanofluid in relation to water are presented in Figure 7.

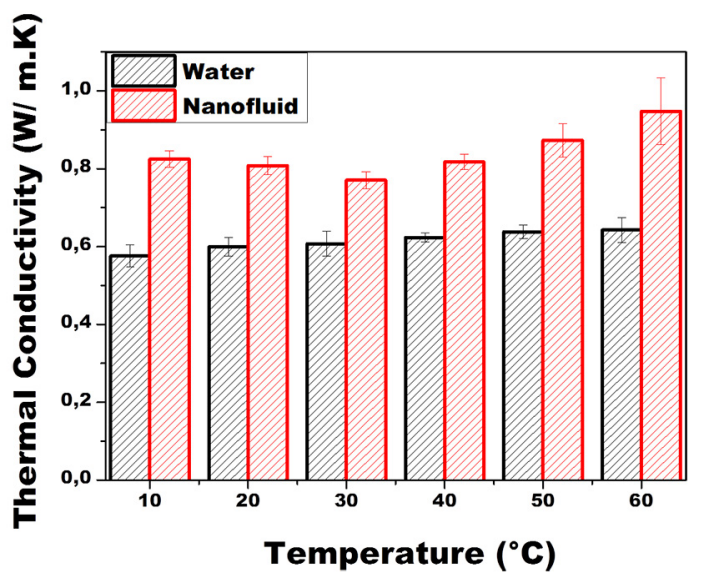

Figure 7. Bar graph of thermal conductivity as a function of the temperature of the nanofluid and distilled water (base fluid)

Analyzing the graph shown in Figure 7, it was observed that the addition of the nanoparticles to the aqueous fluid generated an increase in the thermal conductivity of the fluid. This shows that the nanofluid produced has a better capacity in the heat exchange when compared to the distilled water at all temperatures analyzed, and that a volumetric fraction $0.05 \%$ of $\mathrm{Cu}_{2} \mathrm{~S}$ nanoparticles led to the formation of a nanofluid with thermal conductivity on average $36 \%$ higher than the base fluid. That is three times greater than that of metallic copper with particles of 100 to $200 \mathrm{~nm}$ in the same volumetric fraction ${ }^{26}$, or three times greater than that found by Wilk et al 2017 with nanoparticles of $40 \mathrm{~nm}^{24}$, or even approximately equal to $\mathrm{CuO}$ with a size of $29 \mathrm{~nm}$ in the volumetric fraction of $2 \%{ }^{45}$ The complex nature of the system is dependent on the purity of the nanomaterials of the volumetric fraction and the colloidal stability of the system..$^{5,14,16}$

\section{Conclusion}

Based on the experimental results, it was possible to obtain a nanofluid with high thermal conductivity using a small volumetric fraction of copper sulfide nanoparticles $(\varphi=0.05 \%)$ and to generate an average increase of $36 \%$ in thermal conductivity. The nanoparticles were obtained by the chemical reduction method and were formed by mixing copper sulfide phases, the majority of which consist of $\mathrm{Cu}_{2} \mathrm{~S}$ sulfide. FTIR analysis showed that the nanoparticles are coated with MPA, which allows its dispersion in the base fluid and guarantees its colloidal stability. Transmission micrographs allowed the average size of the nanoparticles to be calculated as $5.2 \pm 1.6 \mathrm{~nm}$. The DLS size was in the range of $30 \mathrm{~nm}$, and the zeta-potential at $\mathrm{pH} 9.0$ was $-25 \mathrm{mV}$, ensuring a good colloidal stability for the nanofluid. The nanoparticles were dispersed in distilled water without the use of high shear rates dispersers. The increase in thermal conductivity of the nanofluid of approximately $36 \%$, combined with its colloidal stability, suggests that the nanofluid produced could be a promising heat exchanger candidate, since even in smaller volumetric fractions, higher increases were obtained in thermal conductivity, when compared to other materials derived from copper.

\section{Acknowledgments}

We would like to thank the funding agencies that allowed the development of the present work, namely the Brazilian agencies CNPq, FAPESP (project 2015/126385), and CAPES. Thanks are due to LNNano for microscopic analysis. We would like to thank the NETeF group of the University of São Paulo, School of Engineering of São Carlos, Department of Mechanical Engineering, São Carlos, SP, Brazil, which allowed us to use the probe for analysis of the thermal conductivity of the nanofluid produced.

\section{References}

1. Lee D, Cheng CC. Energy savings by energy management systems: A review. Renewable and Sustainable Energy Reviews. 2016;56:760-777.

2. Sohel Murshed SM, Nieto de Castro CA. A critical review of traditional and emerging techniques and fluids for electronics cooling. Renewable and Sustainable Energy Reviews. 2017;78:821-833.

3. Sidik NAC, Yazid MNAWM, Mamat R. Recent advancement of nanofluids in engine cooling system. Renewable and Sustainable Energy Reviews. 2017;75:137-144.

4. Ganvir RB, Walke PV, Kriplani VM. Heat transfer characteristics in nanofluid-A review. Renewable and Sustainable Energy Reviews. 2017;75:451-460

5. Timofeeva EV. Nanofluids for Heat Transfer - Potential and Engineering Strategies. In: Ahsan DA, ed. Two Phase Flow, Phase Change and Numerical Modeling. Rijeka: InTech; 2011. p. 435-450.

6. Wong KV, De Leon O. Applications of Nanofluids: Current and Future. Advances in Mechanical Engineering. 2010;2:519659. 
7. Hamzah MH, Sidik NAC, Ken TL, Mamat R, Najafi G. Factors affecting the performance of hybrid nanofluids: A comprehensive review. International Journal of Heat and Mass Transfer. 2017;115(Pt A):630-646.

8. Babu JAR, Kumar KK, Rao SS. State-of-art review on hybrid nanofluids. Renewable and Sustainable Energy Reviews. 2017;77:551-565.

9. Yu W, Xie H, Chen L. Nanofluids. In: Hashim DA, ed. Smart Nanoparticles Technology. Rijeka: InTech; 2012. p. 497-518.

10. Choi SUS. Nanofluid technology: current status and future research. In: Second Korean-American Scientists and Engineers Association Research Trend Study Project Review and the Korea-U.S. Technical Conference on Strategic Technologies; 1988 Oct 22-24; Vienna, VA, USA.

11. Tawfik MM. Experimental studies of nanofluid thermal conductivity enhancement and applications: A review. Renewable and Sustainable Energy Reviews. 2017;75:1239-1253.

12. Angayarkanni SA, Philip J. Review on thermal properties of nanofluids: Recent developments. Advances in Colloid and Interface Science. 2015;225:146-176.

13. Das PK. A review based on the effect and mechanism of thermal conductivity of normal nanofluids and hybrid nanofluids. Journal of Molecular Liquids. 2017;240:420-446.

14. Ghadimi A, Metselaar H, Lotfizadehdehkordi B. Nanofluid Stability optimization based on UV-VIS spectrophotometer measurement. Journal of Engineering Science and Technology. 2015;10:32-40.

15. Herrera AP, Barrera C, Zayas Y, Rinaldi C. Monitoring colloidal stability of polymer-coated magnetic nanoparticles using AC susceptibility measurements. Journal of Colloid and Interface Science. 2010;342(2):540-549.

16. Timofeeva EV, Routbort JL, Singh D. Particle shape effects on thermophysical properties of alumina nanofluids. Journal of Applied Physics. 2009;106(1):014304.

17. Lee GJ, Kim CK, Lee MK, Rhee CK, Kim S, Kim C. Thermal conductivity enhancement of $\mathrm{ZnO}$ nanofluid using a one-step physical method. Thermochimica Acta. 2012; 542:24-27.

18. Phuoc TX, Soong Y, Chyu MK. Synthesis of Ag-deionized water nanofluids using multi-beam laser ablation in liquids. Optics and Lasers in Engineering. 2007;45(12):1099-1106.

19. Deshmukh SS, Sangawar VS. One-step synthesis of polyethylene microspheres using a modified chemical route for pulmonary drug delivery. Journal of Taibah University for Science. 2016;10(4):485-489.

20. Kim HJ, Bang IC, Onoe J. Characteristic stability of bare Auwater nanofluids fabricated by pulsed laser ablation in liquids. Optics and Lasers in Engineering. 2009;47(5):532-538.

21. Yazid MNAWM, Sidik NAC, Mamat R, Najafi G. A review of the impact of preparation on stability of carbon nanotube nanofluids. International Communications in Heat and Mass Transfer. 2016;78:253-263.

22. Wang XQ, Mujumdar AS. A review on nanofluids - part II: experiments and applications. Brazilian Journal of Chemical Engineering. 2008;25(4):631-648.
23. Patil M, Seo JH, Kang SJ, Lee MY. Review on Synthesis, Thermo-Physical Property, and Heat Transfer Mechanism of Nanofluids. Energies. 2016;9(10):840.

24. Wilk J, Smusz R, Grosicki S. Thermophysical properties of water based $\mathrm{Cu}$ nanofluid used in special type of coil heat exchanger. Applied Thermal Engineering. 2017;127:933-943.

25. Xuan Y, Li Q. Heat transfer enhancement of nanofluids. International Journal of Heat and Fluid Flow. 2000;21(1):5864.

26. Liu MS, M Lin MCC, Tsai CY, Wang CC. Enhancement of thermal conductivity with $\mathrm{Cu}$ for nanofluids using chemical reduction method. International Journal of Heat and Mass Transfer. 2006;49(17-18):3028-3033.

27. Wei X, Kong T, Zhu H, Wang L. $\mathrm{CuS} / \mathrm{Cu}_{2} \mathrm{~S}$ nanofluids: Synthesis and thermal conductivity. International Journal of Heat and Mass Transfer. 2010;53(9-10):1841-1843.

28. Pryazhnikov MI, Minakov AV, Rudyak VY, Guzei DV. Thermal conductivity measurements of nanofluids. International Journal of Heat and Mass Transfer. 2017;104:1275-1282.

29. Usman MS, Ibrahim NA, Shameli K, Zainuddin N, Yunus WM. Copper nanoparticles mediated by chitosan: synthesis and characterization via chemical methods. Molecules. 2012;17(12):14928-14936.

30. Li M, Xiang K, Luo G, Gong D, Shen Q, Zhang L. Preparation of Monodispersed Copper Nanoparticles by an Environmentally Friendly Chemical Reduction. Chinese Journal of Chemistry. 2013;31(10):1285-1289.

31. Cheng X, Zhang X, Yin H, Wang A, Xu Y. Modifier effects on chemical reduction synthesis of nanostructured copper. Applied Surface Science. 2006;253(5):2727-2732.

32. Fontes DH, Ribatski G, Bandarra Filho EP. Experimental evaluation of thermal conductivity, viscosity and breakdown voltage $\mathrm{AC}$ of nanofluids of carbon nanotubes and diamond in transformer oil. Diamond and Related Materials. 2015;58:115121.

33. Kumarakuru H, Coombes MJ, Neethling JH, Westraadt JE. Fabrication of $\mathrm{Cu}_{2} \mathrm{~S}$ nanoneedles by self-assembly of nanoparticles via simple wet chemical route. Journal of Alloys and Compounds. 2014;589:67-75.

34. Kim WY, Palve BM, Pathan HM, Joo OS. Spray pyrolytic deposition of polycrystalline $\mathrm{Cu}_{2} \mathrm{~S}$ thin films. Materials Chemistry and Physics. 2011;131(1-2):525-528.

35. Jiménez-Hernández L, Estévez-Hernández $\mathrm{O}$, Hernández-Sánchez M, Díaz JA, Farías-Sánchez M, Reguera E. 3-mercaptopropionic acid surface modification of $\mathrm{Cu}$-doped $\mathrm{ZnO}$ nanoparticles: Their properties and peroxidase conjugation. Colloids and Surfaces A: Physicochemical and Engineering Aspects. 2016;489:351359.

36. Hocaoglu I, Çizmeciyan MN, Erdem R, Ozen C, Kurt A, Sennaroglu A, Acar HY. Development of highly luminescent and cytocompatible near-IR-emitting aqueous Ag2S quantum dots. Journal of Materials Chemistry. 2012;22:14674-14681.

37. Wang M, Niu W, Wu X, Li L, Yang J, Shuang S, et al. Fluorescence enhancement detection of uric acid based on water-soluble 3-mercaptopropionic acid-capped core/shell $\mathrm{ZnS}: \mathrm{Cu} / \mathrm{ZnS}$. RSC Advances. 2014;4(48):25183-25188. 
38. Nakamoto K. Infrared and Raman spectra of inorganic and coordination compounds, Part B: Applications in Coordination, Organometallic, and Bioinorganic. New York: Wiley; 2009.

39. Nafees M, Ikram M, Ali S. Thermal behavior and decomposition of copper sulfide nanomaterial synthesized by aqueous sol method. Digest Journal of Nanomaterials and Biostructures. 2015;10(2):635-641.

40. Fullston D, Fornasiero D, Ralston J. Zeta potential study of the oxidation of copper sulfide minerals. Colloids and Surfaces A: Physicochemical and Engineering Aspects. 1999;146(1-3):113121.

41. Peng Y, Zhao S. The effect of surface oxidation of copper sulfide minerals on clay slime coating in flotation. Minerals Engineering. 2011;24(15):1687-1693.
42. Singh AK. Structure, Synthesis, and Application of Nanoparticles. In: Singh AK. Engineered Nanoparticles - Structure, Properties and Mechanisms of Toxicity. Chapter 2. Boston: Academic Press; 2016. p. 19-76.

43. Kim HJ, Lee SH, Lee JH, Jang SP. Effect of particle shape on suspension stability and thermal conductivities of water-based bohemite alumina nanofluids. Energy. 2015;90(Pt 2):1290-1297.

44. Hwang YJ, Ahn YC, Shin HS, Lee CG, Kim GT, Park HS, et al. Investigation on characteristics of thermal conductivity enhancement of nanofluids. Current Applied Physics. 2006;6(6):1068-1071.

45. Ramesh G, Prabhu NK. Review of thermo-physical properties, wetting and heat transfer characteristics of nanofluids and their applicability in industrial quench heat treatment. Nanoscale Research Letters. 2011;6(1):334. 